\title{
Taking Psychedelics Seriously
}

\author{
Ira Byock, MD, FAAHPM ${ }^{1,2}$
}

\begin{abstract}
Background: Psychiatric research in the 1950s and 1960s showed potential for psychedelic medications to markedly alleviate depression and suffering associated with terminal illness. More recent published studies have demonstrated the safety and efficacy of psilocybin, MDMA, and ketamine when administered in a medically supervised and monitored approach. A single or brief series of sessions often results in substantial and sustained improvement among people with treatment-resistant depression and anxiety, including those with serious medical conditions.

Need and Clinical Considerations: Palliative care clinicians occasionally encounter patients with emotional, existential, or spiritual suffering, which persists despite optimal existing treatments. Such suffering may rob people of a sense that life is worth living. Data from Oregon show that most terminally people who obtain prescriptions to intentionally end their lives are motivated by non-physical suffering. This paper overviews the history of this class of drugs and their therapeutic potential. Clinical cautions, adverse reactions, and important steps related to safe administration of psychedelics are presented, emphasizing careful patient screening, preparation, setting and supervision. Conclusion: Even with an expanding evidence base confirming safety and benefits, political, regulatory, and industry issues impose challenges to the legitimate use of psychedelics. The federal expanded access program and right-to-try laws in multiple states provide precendents for giving terminally ill patients access to medications that have not yet earned FDA approval. Given the prevalence of persistent suffering and growing acceptance of physician-hastened death as a medical response, it is time to revisit the legitimate therapeutic use of psychedelics.
\end{abstract}

Keywords: depression; existential suffering; MDMA; palliative care patients; pharmaco-assisted therapy; posttraumatic stress disorder; psilocybin; psychedelic drugs; therapeutic use

$\mathbf{R}$ ECENTLY PUBLISHED STUDIES in peer-reviewed journals ${ }^{1-4}$ and high-profile articles in the New Yorker, ${ }^{5} \mathrm{New}$ York Times, ${ }^{6}$ and Wall Street Journal, ${ }^{7}$ have rekindled professional and public interest in the therapeutic use of psychedelic drugs. It is easy to understand the enthusiasm. The magazine and newspaper articles include accounts of patients with profound depression, demoralization associated with terminal illness, and anxiety related to post-traumatic stress disorder (PTSD), who experienced remarkable improvements, including some who had previously considered suicide.

Nevertheless, psychiatric and palliative care clinicians who care for profoundly depressed, anxious, and seriously ill patients have every reason to be skeptical. As people become more mentally or physically ill and established treatments remain insufficiently effective, patients' susceptibility increases. Physicians play an important role in protecting vulnerable patients from spurious, nonevidence-based miracle cures, as well as from scientifically grounded, but overly zealous burdensome treatments that are certain to do more harm than good.

An abundance of caution should be accorded psychedelics, which carry real risks and are formally designated Schedule I drugs, signifying that they are dangerous, without therapeutic value, and illegal. Older clinicians remember news stories of deaths of individuals high on hallucinogens who thought they could fly, those with bad trips and flashbacks, and studies that purported to show chromosome damage associated with use of lysergic acid diethylamide (LSD).

However, given the extent of persistent emotional and existential suffering that palliative care clinicians encounter in the patients we serve, these medications deserve serious consideration by our field.

\footnotetext{
${ }^{1}$ Institute for Human Caring, Providence St. Joseph Health, Torrance, California.

${ }^{2}$ Department of Medicine and Community \& Family Medicine, Geisel School of Medicine at Dartmouth, Hanover, New Hampshire. Accepted December 15, 2017.
}

(c) Ira Byock 2018; Published by Mary Ann Liebert, Inc. This Open Access article is distributed under the terms of the Creative Commons Attribution Noncommercial License (http://creativecommons.org/licenses/by-nc/4.0/) which permits any noncommercial use, distribution, and reproduction in any medium, provided the original author(s) and the source are credited. 


\section{Background}

Psychedelic properties of specific plants (mushrooms and cactuses) have been used for centuries by indigenous cultures to induce expanded states of consciousness and spiritual experiences. ${ }^{8-10}$ During the 1950 s and early 1960s, research sponsored by the National Institute of Mental Health demonstrated potential for drugs of this class to markedly alleviate depression and existential suffering among people with cancer. ${ }^{1-13}$ Subsequently, nonmedical use of these drugs and associated political and cultural upheavals resulted in the Schedule I classification, abruptly banning psychedelics from further clinical research and medical use. Although many of the mid-twentieth century clinical trials involved people with terminal conditions, few references to these published studies can be found in the literature of palliative medicine, a young specialty that developed after this period. Over the past 20 years, a few small clinical studies were conducted abroad, mostly in Europe and the United Kingdom. In the United States, over the past decade, with support from the Multidisciplinary Association for Psychedelic Studies and private funders, a few tenacious researchers earned governmental permission to carry out carefully designed trials of pharmacoassisted therapy with psilocybin and 3,4-Methylenedioxymethamphetamine (MDMA), more commonly known by its street names, Ecstasy and Molly.

The recently published research strengthens findings of earlier studies, showing significant efficacy and few adverse effects when these medications are administered as adjuncts to psychotherapy to carefully screened patients, under medical supervision. ${ }^{1-3}$ Three drugs, psilocybin, ketamine, and MDMA, have attracted most of the recent attention. Psilocybin, a naturally occurring drug found in psilocybe mushrooms, has strong and durable benefits for some patients with treatmentresistant depression, and for those with demoralization, anxiety, and depression associated with terminal illness. Ketamine, a Federal Drug Administration (FDA)-approved anesthetic with analgesic and psychedelic properties, has been used offlabel in patients with treatment-resistant depression. In case studies and small clinical series, ketamine has shown notably positive effects. ${ }^{14-16}$ MDMA, a drug synthesized in 1912 as a potential anticoagulant, was later found to have strong psychoactive properties. In the 1970s and early 1980s, psychiatrists who administered MDMA in the context of psychotherapy observed sometimes dramatic improvements in patients suffering from severe, treatment-resistant PTSD. ${ }^{17,18}$

In deciding how to think about these drugs, the distinction between skepticism and cynicism bears examining. Skepticism is warranted, but cynical nonscientific bias can result in therapeutic nihilism. The history of medicine is studded with occasional leaps in progress - consider small pox vaccination, penicillin, and computed tomography scans - that, shortly before they occurred, might have seemed too good to be true. When I graduated from medical school, the idea that duodenal ulcers were caused by bacteria would have been risible; stem cell transplants and gene-editing therapies were the stuff of science fiction. Surprising medical advances humbly remind us to suspend cynicism and that honest inquiry is warranted.

\section{The Need Is Great}

While not only for people who are dying, specialty palliative care teams serve the sickest patients in our health systems and communities. It is, therefore, not surprising that we occasionally encounter incurably ill people whose suffering persists despite all available evidence-based treatments.

In treating pain and other physical distress, established treatment protocols guide escalations of doses and combinations of analgesics and co-analgesic medications. When a patient is dying and physical pain, dyspnea, seizures, or agitated delirium persists and causes intolerable suffering, as a last resort, comfort can reliably be achieved with proportionate sedation. ${ }^{19}$

However, not all suffering is based solely in physical distress. Palliative care clinicians and teams also encounter patients whose misery is rooted in emotional, social, existential, or spiritual distress. Cancer, heart failure, liver failure, and amyotrophic lateral sclerosis (ALS) or motor neuron disease are among the diseases that can result in a progression of personal losses: Of feeling in control. Of taking care of one's self. Of contributing to others. Of enjoyment. Of meaning and purpose. Ultimately, some ill people say they have lost any reason to go on living.

People who are incurably ill and living with progressive disease-related disabilities can experience anxiety, depression, and demoralization. ${ }^{20,21}$ Psychotherapy alone and drug treatments for such syndromes are often insufficient. Medications for depression may take weeks to become effective or prove ineffective. Antidepressants and anxiolytics carry side effects that can include mental slowing and confusion. These adverse effects are particularly common and hazardous in patients with advanced physical illness, who are also at risk of polypharmacy, multidrug interactions, and concomitant disequilibrium and falls. When nonphysical suffering persists despite prudent approaches, published, evidence-based guidelines are limited.

Severe psychological and existential suffering can rob people of feeling that life is worth living. A sense of unending helplessness and hopelessness compels some to consider ending their lives. Suicide rates have risen $24 \%$ over the past two decades and are highest among middle-aged and elderly adults, particularly men who may suffer most from feelings of dependency. ${ }^{22,23}$ Public health data from Oregon show that since implementation of the Death with Dignity Act, the large majority of patients who received prescriptions for lethal drugs were motivated by nonphysical suffering. Current or fear of future pain contributed in just $26.4 \%$ of cases, while loss of autonomy (91.4\%), decreased ability to enjoy life $(89.7 \%)$, and loss of dignity $(77.0 \%)$ most often brought these people to contemplate hastening their deaths. ${ }^{24}$

\section{Exercising Abundance of Caution: Screening, Supervision, Set and Setting}

Prescribed to carefully screened patients, in recommended doses, in the context of professional counseling and supervision, psilocybin and MDMA have proven to be notably safe. They have no tissue toxicity, do not interfere with liver function, have scant drug-drug interactions, and carry no long-term physical effects.

These drugs are not intoxicants in the usual sense. They do not dull the senses or induce sleepiness. On the contrary, sensory perception is intensified and attention is aroused. Although abuse syndromes have been reported, few people become habituated to these drugs.

Adverse physiological effects are few and of short duration, but can be substantial. During the onset of psychedelic 
experiences nausea and vomiting are not unusual. In this first hour or more, visual and spatial orientation are commonly disrupted, which can give rise to anxiety. Sympathetic nervous system arousal may occur both because of fear, and from direct effects of the drugs. Particularly during the initial phase of sessions, psychedelics dissolve barriers between physical senses resulting in synesthesia; touches, smells, and tastes can take on sounds, shapes and colors. Similarly, emotions and thoughts may evoke visual images and sounds. These phenomena explain why the term hallucinogen is often used synonymously with psychedelics to refer to this class of drugs.

Clinicians and researchers familiar with this class of pharmaceuticals emphasize the importance of screening, supervision, and "set and setting."

\section{Screening}

Not every suffering patient is a candidate for therapy involving psychedelic drugs. As a general guideline, people who have cognitive and emotional conditions associated with disorganized or diminished ego strength are not good candidates for pharmaco-assisted therapy with psychedelics. MDMA may represent a partial exception to this exclusion, because it has fewer cognitive and sensory effects and more salutary emotional and interpersonal properties. Contraindications include people with borderline personality disorders or schizophrenic tendencies.

\section{Supervision}

Supervision is necessary for ensuring safety of psychedelic experiences. Short-term psychological effects are profound. If used in unsupervised fashion by unselected and unprepared people, these drugs can be highly dangerous and, in extreme cases, cause death. The sensory effects described above interfere with hand-eye coordination and fine motor function, making operating a vehicle or machinery or even walking in public potentially dangerous. These effects are sufficient to emphasize that professionals who are skilled in managing adverse effects must be present. Most research into pharmacoassisted therapy with psychedelics has by protocol required subjects to remain in a single comfortable room throughout the sessions. In addition to safety, the supervising therapists are able to guide patients through their experiences to optimize the drug's beneficial potential.

\section{Set and setting}

Anthropologists studying traditional use of psychedelics by shamans and indigenous people recognized the influence of expectations and motivation on subjective experience. Since the earliest psychological research into pharmacoassisted therapy with psychedelics, clinicians have emphasized the importance of "set and setting."

The dissolution of assumptions and diminution of barriers caused by these drugs extend to psychological and interpersonal realms of experience. An enhanced sense of connection to others not only underpins some of the therapeutic effects, but also results in vulnerability to emotional contagion. When taken without adequate preparation and when surroundings are anxiety-provoking - either physically uncomfortable or emotionally intimidating - the psychedelic experience predictably results in fear, a prolonged sense of dread, or full panic. Conversely, in controlled settings with elements of soft light, art, and appropriate music, or nature, and gentle, compassionate people, such adverse reactions are rare.

With adequate counseling and preparation, and when psychedelic experiences unfold in calm, aesthetically pleasing environments, they prove beneficial in a high proportion of cases. ${ }^{17}$ In these situations, the healing motivations of both therapists and patients may contribute to therapeutic outcomes.

\section{Therapeutic Effects}

Clinical case studies and research trials describe common patterns of subjective experiences that are associated with therapeutic benefits for people with severe anxiety and depression. As the initial phase of psychedelic experience wanes and people regain familiar barriers between visual, auditory, tactile, olfactory senses, people typically report heightened cognitive clarity and expanded emotional receptivity. Previously unrecognized or unquestioned assumptions related to one's place in the world and relationships to nature, one's physical and social environments become available to being considered anew.

While psychedelic experiences vary significantly from one individual to another, research subjects and people interviewed for journalistic articles commonly express attributes, which include heightened clarity and confidence about their personal values and priorities, and a renewed or enhanced recognition of intrinsic meaning and value of life. People often voice a sense of exhilaration, insight, and strengthened connection to others, as well as a richer sense of relationship with nature or God. ${ }^{25}$ People who take psychedelics with an intention of spiritual introspection often report that the drugs opened windows into deeper realms of existential experience. ${ }^{5,9}$ In safe and supportive environments, these effects typically induce a state of wonder, conceptual frame shift, expanded capacity for love, and an intensified sense of connection. Patients living with medical conditions that had robbed them of hope or reason to live may experience a transformative shift in perspective and experience of inherent meaning, value, and worth.

Not all psychedelics drugs are alike and subcategories have been described. ${ }^{10}$ Drugs, such as psilocybin and LSD, classified as entheogens, ${ }^{26}$ are associated with introspection and new insights, shifts of perspective, and reframing of experience and relationship to others and the world. MDMA is characterized as an empathogen, referring to prominent emotional effects of interpersonal warmth, empathy, and openness. ${ }^{27}$ These properties may underlie the benefits of MDMA in the context of therapy for those suffering from severe PTSD.

For most of these drugs, a single six to eight-hour session or short series of sessions suffices for therapeutic benefit. Alleviation of anxiety and depression may persist for weeks to months and, for some, proves permanent. Exceptions to this treatment pattern include protocols of daily low-dose ketamine for depression ${ }^{15}$ and recent nonmedical reports of daily or every third day micro-dosing of LSD. ${ }^{28,29}$

\section{Political and Regulatory Considerations}

Psychedelic drugs were closely associated with the cultural wars of the 1960s and 1970s when strong political undercurrents contributed to this class of drugs being classified Schedule I. Similarly, MDMA became well known as Ecstasy or Molly, a popular, illicit rave and party drug. ${ }^{7}$ In the 
mid-1980s, despite evidence of MDMA's striking efficacy and relative safety when used therapeutically, the FDA declared MDMA a Schedule I agent. Court rulings challenged that classification; however, in 1998 the FDA reaffirmed and made the Schedule I classification permanent. ${ }^{30}$

The process of renewing clinical research of psychedelics has been long and painstaking. Future efforts to reclassify selected psychedelics, such as psilocybin, as Schedule II drugs, enabling both research and clinical administration will likely meet predictable political resistance. There are compelling reasons, however, to address the expected concerns of opponents and proceed with efforts to reclassify these drugs.

Treatment-resistant depression and anxiety associated with PTSD causes untold suffering and contributes to thousands of deaths each year. A few population health studies suggest that rising suicide rates may in part be due to suicide becoming less shameful and more socially acceptable, lowering barriers for people who feel hopeless. ${ }^{31,32}$ A person with severe depression, who has a coexisting serious, lifethreatening physical condition, may feel that his or her quality of life is not worth living and may forgo arduous, but potentially life-saving treatments. Additionally, nearly one sixth of Americans live in states where physician-hastened death is legal and those with terminal illness may choose this option in absence of alternative sources of relief.

There may be higher ground on which political conservatives and progressives - as well as those on opposing sides of the issue of legalizing physician-hastened death-might build consensus. Given the life-threatening nature of persistent, treatment-resistant depression and PTSD, including among veterans of America's wars, and the rising incidence of suicide, the reclassification of psilocybin and MDMA can be legitimately cast as a right-to-try issue. Right-to-try legislation has been used to provide terminally ill patients access to potentially life-extending medications that have been tested in Phase I trials but are of uncertain benefit. ${ }^{33,34}$ Similarly, the FDA's expanded access or compassionate use provisions may make use of drugs that have not been approved available to patients who are otherwise facing death. ${ }^{35,36}$ By diminishing a desire to die among people with severe depression, anxiety, PTSD, and those with terminal cancers, genetic and neurodegenerative diseases, psychedelics may have greater life-saving effects than other drugs that have earned right-totry and expanded access status. ${ }^{37}$

\section{Business Considerations}

Business models for medical uses of these drugs are not clearly defined. Alleviating persistent depression or PTSD with one or two doses of an inexpensive, un-patentable compound may threaten existing markets for antidepressant and anxiolytic medications. Therefore, it is possible that the pharmaceutical industry may oppose legalization and supervised use of these medications. If so, industry lobbying could complicate regulatory processes needed to research legitimate uses of these drugs.

The way forward may include folding the cost of these medications into professional fees for pharmaco-assisted therapy. ${ }^{38}$ This alternative business model would align well with the therapeutic rationale for requiring psychedelic sessions to be supervised by trained counselors, who are able to control set and setting and capable of preventing and managing any adverse reactions.

\section{Final Thoughts}

Faced with novel therapies with reported clinical benefits that seem too good to be true, skepticism is warranted to protect vulnerable patients from harm. Cynicism, however, may prove more dangerous still. Unscientific bias and nihilistic assumptions can keep effective treatments from people who desperately need them.

Despite the controversial history of psychedelic medications, palliative specialists who care for patients with serious medical conditions and common, difficult-to-treat nonphysical suffering have a duty to explore these hopeful, potentially life-preserving treatments. Against the backdrop of physicianhastened death becoming legal in five states, expanded research of clinical psychedelics must proceed.

In reexamining the use of psychedelics in pharmaco-assisted therapy, we must not allow preconceptions, politics, or puritanism to prevent suffering people, who are now considered helpless and hopeless, from receiving promising, at times life-saving, treatments.

\section{Author Disclosure Statement}

No competing financial interests exist.

\section{References}

1. Griffiths RR, Johnson MW, Carducci MA, et al.: Psilocybin produces substantial and sustained decreases in depression and anxiety in patients with life-threatening cancer: A randomized double-blind trial. J Psychopharmacol 2016; 30:1181-1197.

2. Grob CS, Danforth AL, Chopra GS, et al.: Pilot study of psilocybin treatment for anxiety in patients with advancedstage cancer. Arch Gen Psychiatry 2011;68:71-78.

3. Johnson MW, Griffiths RR: Potential therapeutic effects of psilocybin. Neurotherapeutics 2017;14:734-740.

4. Carhart-Harris RL, Roseman L, Bolstridge M, et al.: Psilocybin for treatment-resistant depression: fMRI-measured brain mechanisms. Sci Rep 2017;7:13187.

5. Pollan M: The Treatment Trip. The New Yorker, February 9, 2015.

6. Bone E: Can Mushrooms Treat Depression? The New York Times. November 29, 2014; Sunday Review.

7. Dean J: How MDMA went from club drug to "Breakthrough Therapy." Wall St J 2017.

8. Grof S: LSD Psychotherapy. Sarasota, FL: Multidisciplinary Association for Psychedelic Studies, 1980.

9. Huxley A: The Doors of Perception. Harper \& Brothers, New York, 1954.

10. Masters REL, Houston J: The Varieties of Psychedelic Experience. Dell Publishing, New York, 1966.

11. Griffths RR, Grob CS: Hallucinogens as medicine. Sci Am 2010;303:76-79.

12. Kast E: LSD and the dying patient. Chic Med Sch Q 1966; 26:80-87.

13. Pahnke WN, Kurland AA, Unger S, et al.: The experimental use of psychedelic (LSD) psychotherapy. JAMA 1970;212:1856-1863.

14. Iglewicz A, Morrison K, Nelesen RA, et al.: Ketamine for the treatment of depression in patients receiving hospice care: A retrospective medical record review of thirty-one cases. Psychosomatics 2015;56:329-337.

15. Irwin SA, Iglewicz A, Nelesen RA, et al.: Daily oral ketamine for the treatment of depression and anxiety in 
patients receiving hospice care: A 28-day open-label proofof-concept trial. J Palliat Med 2013;16:958-965.

16. Swiatek KM, Jordan K, Coffman J.: New use for an old drug: Oral ketamine for treatment-resistant depression. BMJ Case Rep 2016;2016: pii: bcr2016216088.

17. Mithoefer MC, Grob CS, Brewerton TD: Novel psychopharmacological therapies for psychiatric disorders: Psilocybin and MDMA. Lancet Psychiatry 2016;3:481-488.

18. Sessa B: MDMA and PTSD treatment: "PTSD: From novel pathophysiology to innovative therapeutics". Neurosci Lett 2017;649:176-180.

19. Quill TE, Byock IR: Responding to intractable terminal suffering: The role of terminal sedation and voluntary refusal of food and fluids. ACP-ASIM End-of-Life Care Consensus Panel. American College of Physicians-American Society of Internal Medicine. Ann Intern Med 2000;132:408-414.

20. Kissane DW, Clarke DM, Street AF: Demoralization syndrome-A relevant psychiatric diagnosis for palliative care. J Palliat Care 2001;17:12-21.

21. Kissane DW: The relief of existential suffering. Arch Intern Med 2012;172:1501-1505.

22. National Institute of Mental Health: Suicide. 2017. https:// www.nimh.nih.gov/health/statistics/suicide/index.shtml part_153200. (Last accessed October 21, 2017).

23. Prevention AFfS: Suicide Statistics. 2017. https://afsp.org/ about-suicide/suicide-statistics (Last accessed October 19, 2017).

24. Oregon PHD: Death with Dignity Act Annual Reports. 2017. https://public.health.oregon.gov (Last accessed April 12, 2017).

25. Carhart-Harris RL, Erritzoe D, Haijen E, et al.: Psychedelics and connectedness. Psychopharmacology (Berl) 2017 [Epub ahead of print]; DOI: 10.1007/s00213-017-4701-y.

26. Ruck CA, Bigwood J, Staples D, et al.: Entheogens. J Psychedelic Drugs 1979;11:145-146.

27. Wagner MT, Mithoefer MC, Mithoefer AT, et al.: Therapeutic effect of increased openness: Investigating mechanism of action in MDMA-assisted psychotherapy. J Psychopharmacol 2017;31:967-974.

28. Williams A: How LSD Saved One Woman's Marriage. New York Times, January 7, 2017.
29. Waldman A: A Really Good Day: How Microdosing Made a Mega Difference in My Mood, My Marriage, and My Life. Alfred A. Knopf, New York, 2017.

30. Boire RG. The Politics of Medicine: The Scheduling of MDMA. 2000. www.cognitiveliberty.org/dll/mdma_scheduling_history. htm (Last accessed October 8, 2017).

31. Jones DA, Paton D: How does legalization of physicianassisted suicide affect rates of suicide? South Med J 2015; 108:599-604.

32. Kheriaty A: Social contagion effects of physician-assisted suicide: Commentary on "How Does Legalization of Physician-Assisted Suicide Affect Rates of Suicide?". South Med J 2015;108:605-606.

33. Jonhson BE, Heine MJ: What patients should know about Pa.'s new 'right to try' legislation. The Inquirer, October 19, 2017.

34. Simmons Z. Right-to-try investigational therapies for incurable disorders. Continuum (Minneap Minn) 2017;23(5, Peripheral Nerve and Motor Neuron Disorders):1451-1457.

35. Weintraub K: Novel system to get dying patients an experimental cancer drug raises hopes-And thorny questions. STAT News, 2016.

36. Miller JE, Ross JS, Moch KI, Caplan AL: Characterizing expanded access and compassionate use programs for experimental drugs. BMC Res Notes 2017;10:350.

37. Cohen-Kurzrock BA, Cohen PR, Kurzrock R: Health policy: The right to try is embodied in the right to die. Nat Rev Clin Oncol 2016;13:399-400.

38. Goldsmith G: Personal communication, March 2, 2017.

Address correspondence to: Ira Byock, MD, FAAHPM Institute for Human Caring Providence St. Joseph Health 5315 Torrance Boulevard

Suite B-1

Torrance, CA 90503

E-mail: ira.byock@gmail.com 